\title{
Hadron electromagnetic form factors in the space- and timelike regions and higher Fock states
}

\section{Giovanni Salmè*}

Istituto Nazionale di Fisica Nucleare, Sezione di Roma, P.le A. Moro 2, I-00185 Roma, Italy

E-mail: salmeg@romal.infn.it

\section{J. Pacheco B.C de Melo}

Centro de Ciências Exatas e Tecnológicas, Universidade Cruzeiro do Sul, 08060-070, Brazil

\section{Tobias Frederico}

Departamento de Física, Instituto Tecnológico de Aeronáutica, 12.228-900 São José dos

Campos, São Paulo, Brazil

\section{Emanuele Pace}

Dipartimento di Fisica, Università di Roma "Tor Vergata" and Istituto Nazionale di Fisica Nucleare, Sezione Tor Vergata, Via della Ricerca Scientifica 1, I-00133 Roma, Italy

\section{Silvia Pisano}

Institut de Physique Nucléaire Orsay, F-91406 Orsay, France

\begin{abstract}
We briefly review our approach for calculating hadron electromagnetic form factors, both in the spacelike and timelike regions. The main features of our model are: i) a suitable light-front projection of the Mandelstam formula for the matrix elements of the current operator, and ii) a microscopic Vector Meson Dominance model. Outcomes of our approach, like a very peculiar interpretation of the existing puzzle for the ratio $\mu_{p} G_{E}^{p} / G_{M}^{p}$, in the spacelike region, and the relevance of possible missing strengths in the timelike region, are discussed.
\end{abstract}

LIGHT CONE 2008 Relativistic Nuclear and Particle Physics

July 7-11 2008

Mulhouse, France

\footnotetext{
*Speaker.
} 


\section{Introduction}

The investigation of hadron electromagnetic (em) form factors in the spacelike (SL) and timelike (TL) regions, within the light-front (LF) dynamics, opens a unique possibility to study hadronic states, by exploiting the almost simple LF vacuum (see e.g. [1] for a recent review). In view of this, the Fock expansion of hadronic states, like $\mid$ meson $\rangle=|q \bar{q}\rangle_{\text {val }}+\left\{|q \bar{q} q \bar{q}\rangle+|q \bar{q} g\rangle_{\ldots . . .}\right\}_{\text {nonval }}$ and $\mid$ baryon $\rangle=|q q q\rangle_{\text {val }}+\{|q q q q \bar{q}\rangle+|q q q g\rangle \ldots . .\}_{\text {nonval }}$, becomes physically meaningful, with the well known caveat related to the presence of the so-called zero modes, necessary for recovering the chiral symmetry breaking within a LF framework. Moreover, in the TL region, a change of reference frame, i.e. from $q^{+}=0$ to $q^{+} \neq 0$, yields the possibility to address the vast phenomenology of hadronic resonances, e.g. isoscalar (IS) and isovector (IV) Vector Mesons (VM's).

Aim of our approach is to include effects beyond CQM, as suggested by the Fock expansion of a hadron state, taking in great consideration the clue suggested by the present difficulties for relativistic constituent quark models (CQM's) to give a detailed description of the Nucleon form factors (ff's), without implementing new features, like e.g. the quark ff's (see [2] for the first systematic study of those quantities). Furthermore, we have extended the yield of comparison, treating on the same footing both SL and TL em form factors. This improvement imposes a change of reference frame, namely adopting a frame where $\mathbf{q}_{\perp}=0$ and $q^{+} \neq 0$ [3].

\section{Em current and the Mandelstam Formula}

For an interacting system, a covariant expression of the matrix elements of the em current can be obtained is given by the Mandelstam formula [4], that represents the starting point of our approach. For illustrative purpose, let us consider the Pion in the TL region. Then one has [5]

$$
\left\langle P_{\pi} P_{\bar{\pi}}\left|j^{\mu}\right| 0\right\rangle=\mathscr{R} \int \frac{d^{4} k}{(2 \pi)^{4}} \Lambda_{\bar{\pi}}\left(k^{\prime}, P_{\bar{\pi}}\right) \bar{\Lambda}_{\pi}\left(k, P_{\pi}\right) \operatorname{Tr}\left[S\left(k^{\prime}\right) \gamma^{5} S(k-q) \Gamma^{\mu}(k, q) S(k) \gamma^{5}\right]
$$

where $\mathscr{R}=-\imath N_{c} 2 e m^{2} / f_{\pi}^{2}, N_{c}$ is the number of colors, $k^{\prime}=k-P_{\pi}, S(p)=1 /(\not p-m+\imath \varepsilon)$ is the CQ propagator, $\gamma_{5} \Lambda_{\pi}\left(k, P_{\pi}\right)$ the Pion vertex function (deduced from a simple effective quark-Pion Lagrangian), $P_{\pi}^{\mu}$ and $P_{\bar{\pi}}^{\mu}$ the Pion momenta, $\Gamma^{\mu}(k, q)$ the quark-photon vertex and $q^{\mu}$ the virtual photon momentum. The corresponding expression for the SL case can be obtained by standard changes [5].

The next step is represented by the integration over $k^{-}$, that realizes a projection of the Mandelstam formula onto the 3D LF hyperplane. If we knew the whole analytic structure of both the Pion vertex and the quark-photon vertex, then the integration could be done exactly, but in absence of such detailed information, one should apply some approximation, like considering only the poles of the propagators. This approximation turns out to be a very effective one in the $q^{+} \neq 0$ frame [6]. Once, the integration is performed under the above approximation, one can easily singled out the expected contributions, both in the SL region (i.e. the triangle contribution and the $q \bar{q}$-production term or Z-diagram) and in the TL region, where the photon materializes in a $q \bar{q}$ pair, ( $\operatorname{cf}[5])$.

The analytic approximation generates two new questions that we solved through phenomenological assumptions. The first one is: how to model the quark-photon vertex, and the second one is: how to describe both the $q \bar{q}$-Pion vertex in the valence sector and the emission or absorption of a Pion by a quark, that leads to the non valence vertex. 
A simplification in the calculation can be achieved by a quite natural assumption, namely a vanishing Pion mass. Then, both in TL and SL regions, only diagrams with a $q \bar{q}$ production contribute. In view of this, it is necessary to model a Vector Meson Dominance (VMD) approach for describing the quark-photon vertex (without a bare term, still due to the vanishing Pion mass), viz

$$
\Gamma^{\mu}(k, q)=\sqrt{2} \sum_{n, \lambda}\left[\varepsilon_{\lambda} \cdot \widehat{V}_{n}\left(k, k-P_{n}\right)\right] \Lambda_{n}\left(k, P_{n}\right) \frac{\left[\varepsilon_{\lambda}^{\mu}\right]^{*} f_{V n}}{\left(q^{2}-M_{n}^{2}+\imath M_{n} \tilde{\Gamma}_{n}\left(q^{2}\right)\right)}
$$

where $f_{V n}$ is the decay constant of the n-th VM into a virtual photon (to be calculated in our model), while $M_{n}$ is the mass, $\tilde{\Gamma}_{n}\left(q^{2}\right)=\Gamma_{n} q^{2} / M_{n}^{2}$ (for $q^{2}>0$ ) the corresponding total decay width and $\varepsilon_{\lambda}\left(P_{n}\right)$ the VM polarization. Moreover, $\left[\varepsilon_{\lambda}\left(P_{n}\right) \cdot \widehat{V}_{n}\left(k, k-P_{n}\right)\right] \Lambda_{n}\left(k, P_{n}\right)$ is the VM vertex function. The Dirac structure, $\widehat{V}_{n}^{\mu}\left(k, k-P_{n}\right)$, is chosen in order to generate the proper Melosh rotations for ${ }^{3} S_{1}$ states [7], while $\Lambda_{n}(k, q)$, the momentum-dependent part of the VM Bethe-Salpeter amplitude, will be approximated on the LF hyperplane as follows.

In the valence sector, 3D amplitudes are described through LF VM wave functions [5], i.e.

$$
\frac{\left.P_{n}^{+} \Lambda_{n}\left(k, P_{n}\right)\right|_{\left[k^{-}=k_{o n}^{-}\right]}}{\left[M_{n}^{2}-M_{0}^{2}\left(k^{+}, \mathbf{k}_{\perp} ; P_{n}^{+}, \mathbf{P}_{n \perp}\right)\right]}=\psi_{n}\left(k^{+}, \mathbf{k}_{\perp} ; P_{n}^{+}, \mathbf{P}_{n \perp}\right)
$$

where $k_{o n}^{-}=\left(m^{2}+|\mathbf{k}|_{\perp}^{2}\right) / k^{+}$, and $\psi_{n}\left(k^{+}, \mathbf{k}_{\perp} ; P_{n}^{+}, \mathbf{P}_{n \perp}\right)$ is an eigenfunction of the relativistic CQ square mass operator of Ref. [11], with both confinement (harmonic oscillator potential) and $\pi-\rho$ splitting (Dirac-delta interaction in the pseudoscalar channel). Moreover, it is normalized to the probability of the valence Fock state, according to the model elaborated in [5]. As to the valence component of the Pion, an analogous Ansatz has been adopted. Then, two different calculations have been generated by using the Pion eigenstate of the model in Ref. [11] and the pQCD asymptotic wave function.

In the non valence region, besides the Pion valence component there is the non valence component, given by the emission (absorption) of a Pion by a quark. We assume a constant interaction for describing such a process, like in Ref. [12]. Note that the coupling constant is fixed by the normalization of the Pion ff.

Finally, as a consequence of the simplifying assumption $m_{\pi}=0$, the SL triangle term is vanishing and, notably, in the pair-production terms one has only instantaneous contributions, produced by the standard LF decomposition of the Dirac propagator (i.e. $S(k)=\left(k_{\text {on }}+m\right) /\left[k^{+}\left(k^{-}-k_{o n}^{-}+\right.\right.$ $i \varepsilon)]+\gamma^{+} / 2 k^{+}$). In order to model instantaneous vertex functions entering in such contributions, we put $\Lambda^{i s t} \sim \mathscr{C} \Lambda^{\text {full }}$, where the constant $\mathscr{C}$ is thought to roughly describe the effects of the short-range interaction. Indeed, we use the relative weight, $w_{V M}=\mathscr{C}_{V M} / \mathscr{C}_{\pi}$, as a free parameter.

\section{Pion em Form Factor in the SL and TL regions}

The microscopic calculation of the Pion em $\mathrm{ff}$ can be realized by applying the previous ingredients, and the results are shown in Fig. 1, where only the IV VM's are considered and the CQ mass is $m_{q}=200 \mathrm{MeV}$, (see also [5] where $m_{q}=265 \mathrm{MeV}$ was adopted). In [5], experimental IV VM masses and widths for the first four resonances have been used, while for the other IV VM (up 


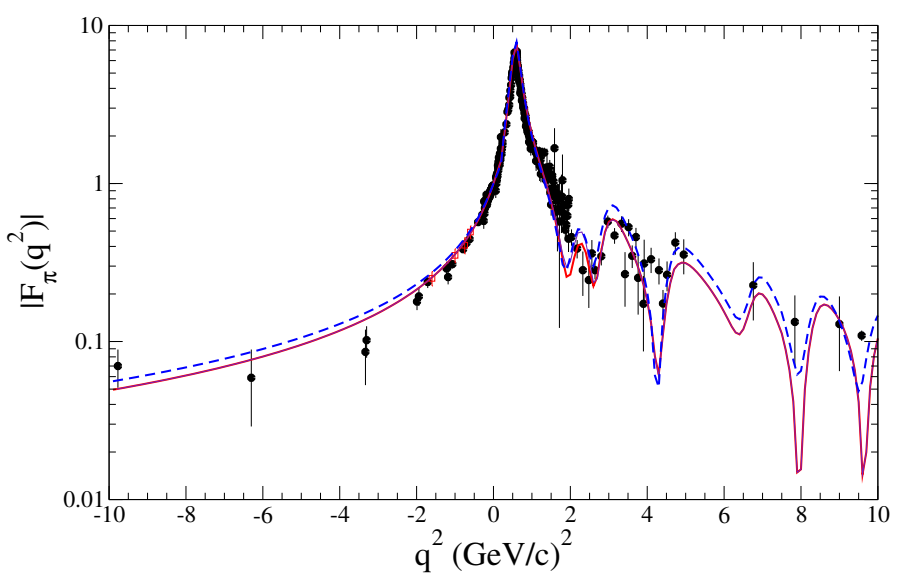

Figure 1: The Pion em form factor vs $q^{2}$. Theoretical calculations with two free parameters: i) IV VM widths for $n>4, \Gamma_{n>4}=0.15 \mathrm{GeV}$, ii) the constant $w_{V M}=-1.0$ for describing the instantaneous vertexes (see text). Solid line: calculation with the Pion wave function from the model of [11]; dashed line: the same as the solid line, but with the asymptotic Pion wave function (see [5]). Data from the compilation of Ref. [8] and from the TJLAB collaboration [9]. (Adapted from [5]).

to $n=20$ in Eq. (2.2)), calculations based on the eigenfunctions of Ref. [11] have been exploited. The two free parameters present in our VMD microscopical approach are: i) the VM width for $n>4$, taken equal to $\Gamma_{n>4}=0.150 \mathrm{GeV}$, as suggested by the experimental VM widths around $M_{n} \sim 2 \mathrm{GeV}$, and ii) $w_{V M}=\mathscr{C}_{V M} / \mathscr{C}_{\pi}$, the relative weight of the two instantaneous contributions, taken equal to $w_{V M}=-1.0$, from a global fit. The comparison with the data is quite accurate over a wide kinematical range, but a more refined description of the instantaneous contribution could help in filling the deep near $2(\mathrm{GeV} / \mathrm{c})^{2}$, as well as contributions from $\omega$ - and $\phi$-like mesons. It is worth noting the missing strength around $q^{2} \sim 4(\mathrm{GeV} / \mathrm{c})^{2}$, as in the TL proton ff (cf Fig. 3).

\section{Nucleon em Form Factors in the SL and TL regions}

The Dirac structure of the quark-Nucleon vertex is suggested, as in the case of the quarkPion vertex, by an effective Lagrangian. In the actual calculation the model of Ref. [13] has been adopted, but without a derivative coupling (i.e. putting $\alpha=1$ in the Lagrangian of [13]). The Nucleon vertex function, with a properly symmetrized Dirac structure, is given by[10]

$$
\begin{aligned}
& \Phi_{N}^{\sigma}\left(k_{1}, k_{2}, k_{3}, P_{N}\right)=\imath\left[S\left(k_{1}\right) \tau_{y} \gamma^{5} S_{C}\left(k_{2}\right) C \otimes S\left(k_{3}\right)+S\left(k_{3}\right) \tau_{y} \gamma^{5} S_{C}\left(k_{1}\right) C \otimes S\left(k_{2}\right)+\right. \\
& \left.+S\left(k_{3}\right) \tau_{y} \gamma^{5} S_{C}\left(k_{2}\right) C \otimes S\left(k_{1}\right)\right] \Lambda\left(k_{1}, k_{2}, k_{3}\right) \chi_{\tau_{N}} U_{N}\left(P_{N}, \sigma\right)
\end{aligned}
$$

where $U_{N}$ and $\chi_{\tau_{N}}$ are the nucleon spinor and isospin eigenstate respectively, and $\Lambda$ describes the symmetric momentum dependence of the vertex function upon the quark momenta, $k_{i}$.

For instance, SL em ff's, that are present in the matrix elements of the macroscopic current, can be evaluated microscopically by means of the Mandelstam formula, i.e.

$$
\begin{aligned}
& \left\langle\sigma^{\prime}, P_{N}^{\prime}\left|j^{\mu}\right| P_{N}, \sigma\right\rangle=\bar{U}_{N}\left(P_{N}^{\prime}, \sigma^{\prime}\right)\left[-F_{2}\left(Q^{2}\right) \frac{P_{N}^{\prime}{ }^{\mu}+P_{N}{ }^{\mu}}{2 M_{N}}+G_{M}\left(Q^{2}\right) \gamma^{\mu}\right] U_{N}\left(P_{N}, \sigma\right)=3 N_{c} \times \\
& \int \frac{d^{4} k_{1}}{(2 \pi)^{4}} \int \frac{d^{4} k_{2}}{(2 \pi)^{4}} \sum\left\{\bar{\Phi}_{N}^{\sigma^{\prime}}\left(k_{1}, k_{2}, k_{3}^{\prime}, P_{N}^{\prime}\right) S^{-1}\left(k_{1}\right) S^{-1}\left(k_{2}\right) \mathscr{I}^{\mu}\left(k_{3}, q\right) \Phi_{N}^{\sigma}\left(k_{1}, k_{2}, k_{3}, P_{N}\right)\right\}(4.2)
\end{aligned}
$$


where $\mathscr{I}^{\mu}\left(k_{3}, q\right)$ is the quark-photon vertex and the sum runs over isospin and Dirac indexes. In the TL region, an analogous expression holds [10].

In the frame where $\mathbf{q}_{\perp}=0$ and $q^{+}=\sqrt{\left|q^{2}\right|}$, we integrate on $k_{1}^{-}$and on $k_{2}^{-}$taking into account only the propagator poles, as for the Pion. Then we are left with a 3-momentum dependence of the vertex functions, and diagrammatic contributions in analogy to the Pion (see [10] ).

For the Nucleon case, the quark-photon vertex necessarily contains both isovector and isoscalar terms, $\mathscr{I}^{\mu}=\mathscr{I}_{I S}^{\mu}+\tau_{z} \mathscr{I}_{I V}^{\mu}$, where each term has a purely valence contribution (in the SL region only) and a contribution corresponding to the pair production (or Z-diagram). In turn, the Z-diagram contribution can be decomposed in a bare term + a VMD term (according to the decomposition of the photon state in bare, hadronic [and leptonic] contributions), viz

$$
\mathscr{I}_{i}^{\mu}(k, q)=\mathscr{N}_{i} \theta\left(P_{N}^{+}-k^{+}\right) \theta\left(k^{+}\right) \gamma^{\mu}+\theta\left(q^{+}+k^{+}\right) \theta\left(-k^{+}\right)\left\{Z_{B} \mathscr{N}_{i} \gamma^{\mu}+Z_{V M}^{i} \Gamma_{i}^{\mu}(k, q)\right\}(4.3
$$

with $\mathrm{i}=\mathrm{IS}, \mathrm{IV}, \mathscr{N}_{I S}=1 / 6$ and $\mathscr{N}_{I V}=1 / 2$. The constants $Z_{B}$ (bare term) and $Z_{V M}^{i}$ (VMD term) are unknown weights to be extracted from the phenomenological analysis of the data. Notably, the VMD term $\Gamma_{i}^{\mu}(k, q)$ is the same already used in the Pion case, but now includes IS VM. Up to 20 IS and IV VM have been considered. As in the case of the Pion, $m_{q}=200 \mathrm{MeV}$.

The momentum dependence of the Nucleon vertex function has been modeled in accord to the valence and non valence classification. In the valence sector, the spectator quarks are on their-own $k^{-}$-shell, and the 3-momentum dependence, is approximated through a Nucleon wave function a la Brodsky (PQCD inspired) [1], namely

$$
\frac{\Lambda_{V}\left(k_{1}, k_{2}, k_{3}\right)}{\left[M_{N}^{2}-M_{0}^{2}(1,2,3)\right]}=\mathscr{N} \frac{\left(9 m^{2}\right)^{7 / 2}}{\left(\xi_{1} \xi_{2} \xi_{3}\right)^{p}\left[\beta^{2}+M_{0}^{2}(1,2,3)\right]^{7 / 2}}
$$

where $M_{0}(1,2,3)$ is the free mass of the three-quark system, $\xi_{i}=k_{i}^{+} / P_{N}^{+}$and $\mathscr{N}$ a normalization constant, fixed through the proton charge. The power $7 / 2$ and the parameter $p=0.13$ are chosen for obtaining an asymptotic decrease of the triangle contribution faster than the dipole. Only the triangle diagram determines the magnetic moments and it is very weakly dependent on $p$. Then $\beta=0.65 \mathrm{GeV}$ can be fixed through the evaluation of the anomalous magnetic moments, $\mu_{p}$ and $\mu_{n}$, obtaining for those quantities, $\mu_{p}=2.87\left(\right.$ Exp.=2.793) and $\mu_{n}=-1.85($ Exp. $=-1.913)$.

The non valence vertex can depend on the available invariants, e.g. in the SL region on i) the free mass of the spectator quarks, $M_{0}(1,2)$, and ii) the free mass of the $\mathrm{N}-\bar{q}$ system $M_{0}(N, \overline{3})$. Then, in the SL region we approximate the 3-momentum dependence of the non valence vertex by

$$
\Lambda_{N V}^{S L}\left(k_{1}, k_{2}, k_{3}\right)=\left[g_{12}\right]^{2}\left[g_{N \overline{3}}\right]^{7 / 2-2}\left[\frac{k_{12}^{+}}{P_{N}^{\prime+}}\right]\left[\frac{P_{N}^{+}}{k_{\overline{3}}^{+}}\right]^{r}\left[\frac{P_{N}^{\prime+}}{k_{\overline{3}}^{+}}\right]^{r}
$$

where $k_{12}^{+}=k_{1}^{+}+k_{2}^{+}$and $g_{A B}=\left(m_{A} m_{B}\right) /\left[\beta^{2}+M_{0}^{2}(A, B)\right]$. In the TL region, the non valence vertex can depend on the mass of a Nucleon - $q q$ system (see [10] for more details).

The adjusted parameters are four: i) two weights out three present in the pair production term of Eq. (4.3), i.e. $Z_{B}=Z_{V M}^{I V}=2.283$ and $Z_{V M}^{I S} / Z_{V M}^{I V}=1.12$; ii) the power $p=0.13$ in the valence amplitude, Eq. (4.4); iii) the power $r=0.17$ in the SL and TL non valence vertexes, see e.g. Eq. (4.5). The minimization yields $\chi^{2}=1.7$. Moreover, the calculated proton charge radius is $r_{p}=$ $(0.903 \pm 0.004) f m\left(r_{p}^{e x p}=(0.895 \pm 0.018) f m\right)$, while for the neutron one has $-d G_{E}^{n}\left(q^{2}\right) / d q^{2}=$ 

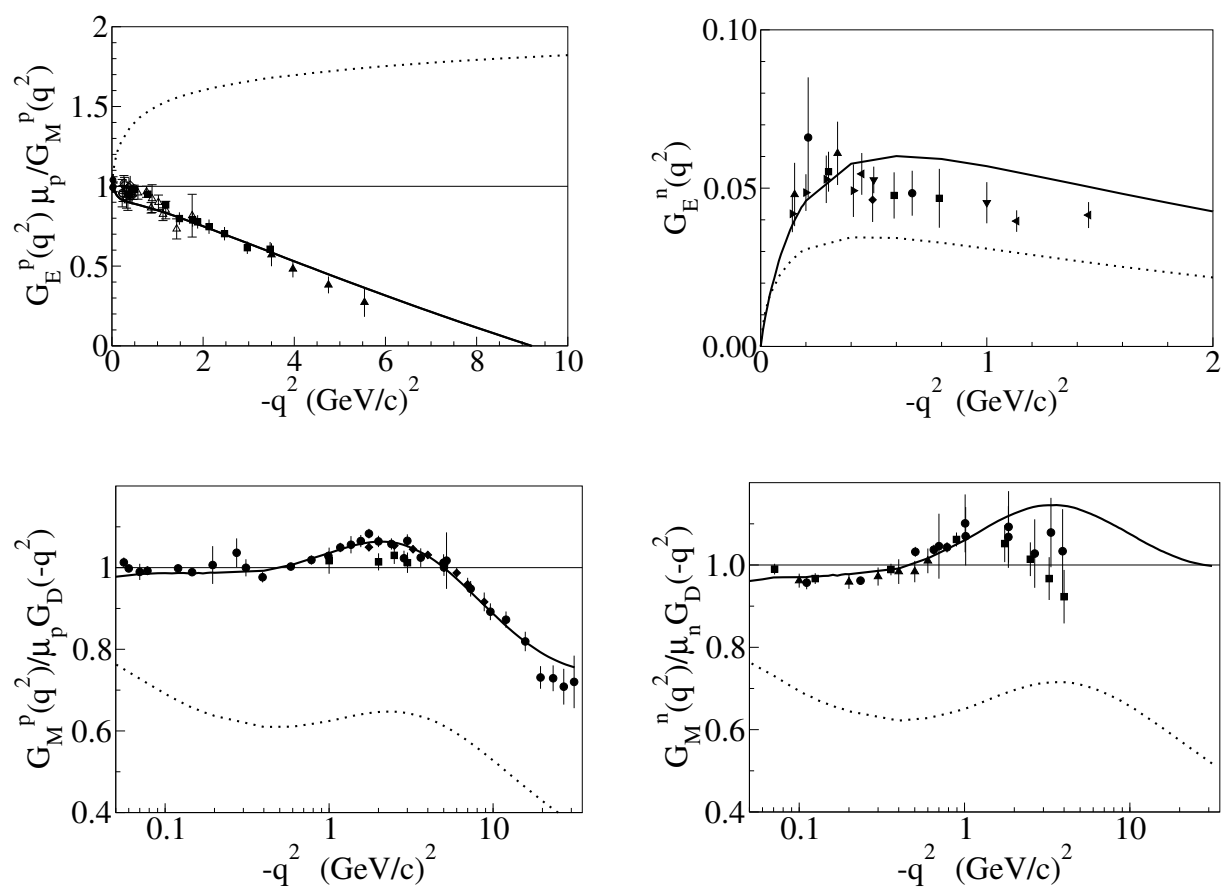

Figure 2: Proton ff's vs $-q^{2}$. Solid line: full calculation; Dotted line: triangle contribution only, i.e. without pair production. Data from the compilation in [14]. $G_{D}\left(\left|q^{2}\right|\right)=\left[1+\left|q^{2}\right| /\left(0.71(\mathrm{GeV} / \mathrm{c})^{2}\right)\right]^{-2}$. (After [10]).

$(0.501 \pm 0.002)(\mathrm{GeV} / \mathrm{c})^{-2}\left(\exp\right.$ value; $-d G_{E}^{n}\left(q^{2}\right) / d q^{2}=(0.512 \pm 0.013)(\mathrm{GeV} / \mathrm{c})^{-2}$. In Fig. 2 the SL nucleon form factors are shown. It is very important to note, that within our approach, the possible zero in $\mu_{p} G_{E}^{p} / G_{M}^{p}$, (at this moment, only suggested by the experimental data), is strongly related to the Z-diagram contribution, i.e. to higher Fock components. Therefore, such a feature, if experimentally confirmed, should represent a clear signature of effects beyond the CQM, since the pair-production contribution is essential for obtaining the vanishing result.

The TL effective form factor, defined by $\left.G_{\text {eff }}\left(q^{2}\right)=\sqrt{\left(\left|G_{M}\left(q^{2}\right)\right|^{2}+\eta\left|G_{E}\left(q^{2}\right)\right|^{2}\right) /(1+\eta}\right)$ (with $\eta=2 m_{N}^{2} / q^{2}$ ), is shown in Fig. 3. It is very interesting to note that a comparison between a theoretical VMD, based on the present-day VM spectra, and the experimental results, could give valuable information. For instance, missing strengths at $q^{2}=4.5(\mathrm{GeV} / \mathrm{c})^{2}$ (the same feature appears in the Pion ff) and $q^{2}=8(\mathrm{GeV} / \mathrm{c})^{2}$, points to an enrichment of the present VM database.

\section{Conclusions}

A microscopical model for Pion and Nucleon em form factors in both SL and TL region has been proposed. The main ingredients are: i) a LF projection of the so-called Mandelstam formula for the matrix elements of the current and ii) a microscopical model for the quark-photon vertex, containing a VMD contribution and, in the Nucleon case, a bare term. The Z-diagram, related to higher Fock components of both hadrons and photon, is essential for both Pion and Nucleon, in our reference frame $q^{+} \neq 0$. In particular, the calculation for the Pion ff, with two free parameters, is in a reasonable agreement with the TL data, and becomes very accurate in the SL region. As to the Nucleon, where we adopted four adjusted parameters, a nice agreement is obtained in the SL 

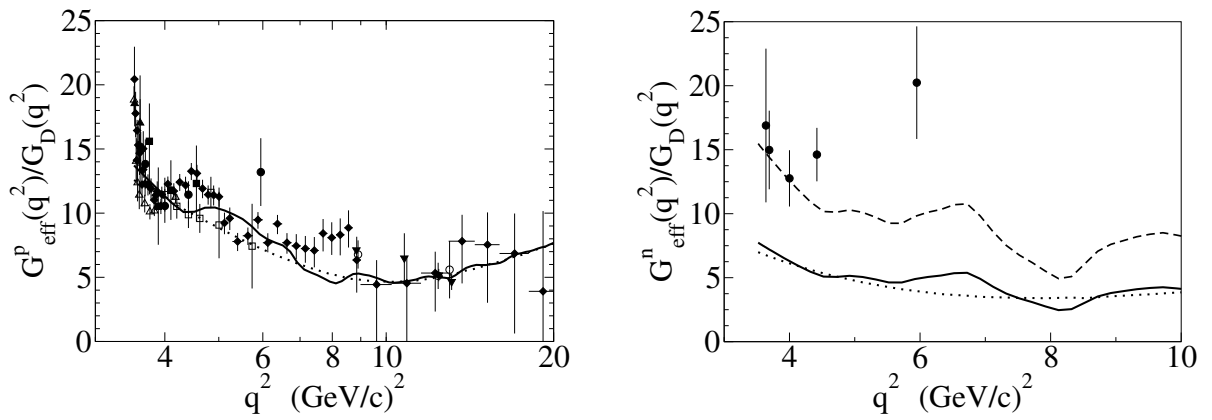

Figure 3: Nucleon effective form factors in the TL region. Left panel: proton ff (data from [15]). Right panel: neutron $\mathrm{ff}$ (data from [16]). Solid line: full calculation; dotted line: bare term only. Dashed line: neutron full calculation multiplied by a factor of two. (After Ref. [10]).

region, with a very interesting interpretation of the possible zero in $\mu_{p} G_{E}^{p} / G_{M}^{p}$, as an effect of a cancellation between the triangle contribution and the pair-production term. In the TL region, the comparison with the proton data is very instructive, since a missing strength for $q^{2}=4.5(\mathrm{GeV} / \mathrm{c})^{2}$ and $q^{2}=8(\mathrm{GeV} / \mathrm{c})^{2}$ indicates possible new resonances. Unfortunately, the available TL neutron [16] data are largely underestimated by the present model.

\section{References}

[1] S.J. Brodsky, H.C. Pauli, and S.S. Pinsky, Phys. Rep. 301, 299 (1998).

[2] F. Cardarelli, E. Pace, S. Simula and G. Salmè, Phys. Lett. B 332, 1 (1994).

[3] E. Pace, F. Lev and G. Salmè, Nucl. Phys. A 641 (1998) 229.

[4] S. Mandelstam, Proc. Royal Soc. A 233, 248 (1955).

[5] J.P.B.C. de Melo, T. Frederico, E. Pace and G. Salmè, Phys. Lett. B 581, 75 (2004); Phys. Rev. D 73, 074013 (2006); Nucl. Phys. A 707, 399 (2002).

[6] J.P.B.C. de Melo , J. S. Veiga , T. Frederico, E. Pace, G. Salmé, hep-ph/0609212.

[7] W. Jaus, Phys. Rev. D 41, 3394(1990).

[8] R. Baldini et al , Eur. Phys. Jou. C 11, 709 (1999), and references therein quoted.

[9] J. Volmer et al, Phys. Rev. Lett. 86, 1713 (2001).

[10] J.P.B.C. de Melo, T. Frederico, E. Pace, S. Pisano and G. Salmè, Nucl. Phys. A 790, 606 (2007) and hep-ph 08041511.

[11] T. Frederico, H.-C. Pauli and S.-G. Zhou, Phys. Rev. D 66, 054007 (2002); Phys. Rev. D 66, 116011 (2002).

[12] C.-R. Ji and H.-M. Choi, Phys. Lett. B 513, 330 (2001).

[13] W.R.B. de Araújo, E.F. Suisso, T. Frederico, M. Beyer and H.J. Weber,, Phys. Lett. B 478, 86 (2001).

[14] C. E. Hyde-Wright and K. de Jager, Ann. Rev. Nucl. and Part. Sci. 54, 217 (2004); C.F. Perdrisat, V. Punjabi, M. Vanderhaeghen, Progr. Part. Nucl. Phys. 59, 694 (2007).

[15] BaBar Collaboration, Phys. Rev. D 73, 012005 (2006), and references therein quoted.

[16] A. Antonelli et al, Nucl. Phys. B 517, 3 (1998). 